\title{
Analyst
}

CORRECTION

(a) CrossMark

Cite this: Analyst, 2016, 141, 1154

DOI: 10.1039/c6an90007k

www.rsc.org/analyst

\section{Correction: Testing and validating electroanalytical simulations}

\author{
Enno Kätelhön and Richard G. Compton*
}

Correction for 'Testing and validating electroanalytical simulations' by Enno Kätelhön and Richard

G. Compton, Analyst, 2015, 140, 2592-2598.

Two brackets were misplaced in the Butler-Volmer equation [eqn (4)], which is correctly given by:

$$
j=k_{A, B}^{0} \exp \left(\frac{-\alpha F\left(E-E_{\mathrm{f}}^{0}\right)}{R T}\right) c_{A}(x=0)-k_{A, B}^{0} \exp \left(\frac{+\beta F\left(E-E_{\mathrm{f}}^{0}\right)}{R T}\right) c_{B}(x=0)
$$

In eqn (16), we provide an expression for the half-wave potential found in a voltammogram recorded for an $n$-electron transfer under Nernstian conditions and an $m: q$ stoichiometry at a microdisk electrode. The expression for the parameter $\gamma$ should be:

$$
\gamma=\frac{D_{\mathrm{A}}}{D_{\mathrm{B}}}
$$

The Royal Society of Chemistry apologises for these errors and any consequent inconvenience to authors and readers. 